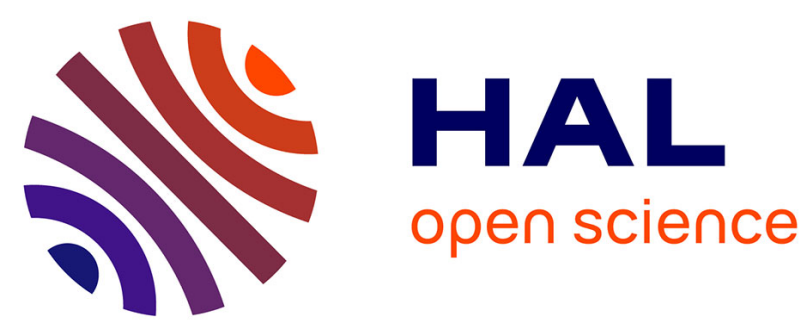

\title{
Interactions modèles expériences sur des âmes nids d'abeilles Nomex (R) : application au design d'un voilier multicoque de course océanique
}

\author{
Laurent Gornet, Gilles Marckmann, Gilles Ollier
}

\section{- To cite this version:}

Laurent Gornet, Gilles Marckmann, Gilles Ollier. Interactions modèles expériences sur des âmes nids d'abeilles Nomex (R) : application au design d'un voilier multicoque de course océanique. Revue des composites et des matériaux avancés = Journal of Composite and Advanced Materials, 2006, Revue des composites et des matériaux avancés, 16 (2), pp.167-190. 10.3166/rcma.16.167-190 . hal-01008352

\section{HAL Id: hal-01008352 \\ https://hal.science/hal-01008352}

Submitted on 6 Feb 2018

HAL is a multi-disciplinary open access archive for the deposit and dissemination of scientific research documents, whether they are published or not. The documents may come from teaching and research institutions in France or abroad, or from public or private research centers.
L'archive ouverte pluridisciplinaire HAL, est destinée au dépôt et à la diffusion de documents scientifiques de niveau recherche, publiés ou non, émanant des établissements d'enseignement et de recherche français ou étrangers, des laboratoires publics ou privés. 


\title{
Interactions modèles expériences sur des âmes nids d'abeilles Nomex®
}

\section{Application au design d'un voilier multicoque de course océanique}

\author{
Laurent Gornet* - Gilles Marckmann*- Gilles Ollier ${ }^{* *}$ \\ *École Centrale de Nantes, Institut de recherche en Génie Civil et Mécanique, GeM, \\ 1 Rue de la Nö̈, BP 92101, 44321 Nantes Cedex 3, France \\ Laurent.gornet@ec-nantes.fr, gilles.marckmann@ec-nantes.fr \\ *MULPTIPLAST, Le Parc du Golf, 56000 VANNES France \\ www.multiplast-yachts.com
}

\begin{abstract}
RÉSUMÉ. Le présent travail concerne la détermination des propriétés mécaniques des âmes nids d'abeilles de forme hexagonale et de leurs versions sur-expansées rectangulaires. Ces âmes en papiers Nomex® sont présentes sur les voiliers multicoques de courses océaniques entièrement réalisés à partir de sandwichs à peaux de tissus carbones. Les caractéristiques mécaniques élastiques de ces âmes sont déterminées à l'aide de la théorie de l'homogénéisation des milieux périodiques. L'étude des modes de flambement du Volume Elémentaire Représentatif permet de déterminer les contraintes ultimes de rupture de l'âme homogène équivalente. La théorie de l'homogénéisation périodique a été implantée dans le code de calcul Eléments Finis Cast3M-CEA. Les propriétés mécaniques et les limites à rupture des âmes en nids d'abeilles sont des données classiquement utilisées lors de la phase de conception et de validation des catamarans océaniques.
\end{abstract}

ABSTRACT. The present study concern the determination of material properties of the hexagonal and rectangular over-expanded honeycombs. These cores made of Nomex® paper are extensively used in the sandwich structures with carbon skins of oceanic multihull sailing race boats. The elastic mechanical properties are determined by periodic homogenization techniques. Finally, RVE buckling modes conduct to determine the ultimate stress of the homogenized material. Periodic homogenized techniques developed in this study are implemented in the finite element code Cast3M-CEA. The honeycomb mechanical properties and ultimate stresses are used to design Oceanic Catamaran structures

MOTS-CLÉS : Structures composites, Nids d'abeilles, âmes Nomex, Eléments Finis, Rupture, homogénéisation périodique.

KEYWORDS: Composite structures, Honeycombs, Nomex cores, Finite element method, Fracture, periodic homogenization. 


\section{Introduction}

Cette étude vise à maîtriser la modélisation et la simulation du comportement mécanique des âmes nids d'abeilles Nomex® utilisées dans la réalisation des voiliers océaniques multicoques de type catamaran. Les dimensions des catamarans de course sont passées en 16 ans de $22 \mathrm{~m}$ pour le Jet Service V (1988) à 37,80m pour le « code B1 » en (2004). En 2000, le catamaran « code 0 » possédait une longueur de 33 m. En 2004, « code B1 », le dernier né du chantier Multiplast atteint la dimension de 37,80m. La plate-forme «code B1» est une structure complexe dont la conception repose sur une technologie de pointe issue de l'aéronautique. Ces catamarans sont réalisés en étuve à partir de formes gauches constituées de structures sandwiches à peaux de carbone époxy. La vérification et l'optimisation de l'échantillonnage du voilier est réalisée à l'aide de simulations éléments finis menées en étroite collaboration avec l'ensemble des acteurs industriels (Figure 1) (Gornet et Marckmann, 2003, The Cata, 2003, Delhommeau, 1988).

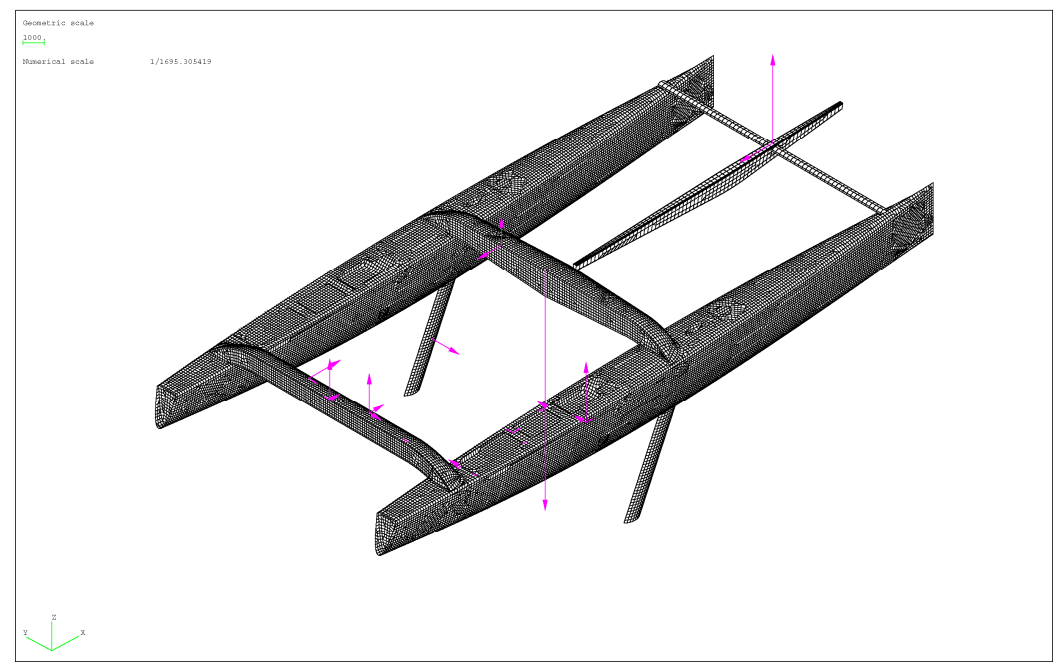

Figure 1. Maillage coque du catamaran code B1, avec son chargement dans la houle

Le niveau des compétitions impose un allégement des structures afin de conserver un bon rendement entre le poids et la puissance vélique de ces maxicatamaran. Lors des grandes courses océaniques, comme The Race, les conditions atmosphériques extrêmes et le haut niveau des compétitions entraînent parfois les concurrents à faire des escales pour réparer des avaries structurelles. Les âmes en nids d'abeilles constituant les bordés et les carénages des voiliers sont parfois incriminés pour leur manque de durabilité à l'impact répéter des vagues et en conséquence, le Design Team se doit de minimiser ce phénomène. Les essais 
mécaniques de validation des peaux en carbone sont réalisés afin d'utiliser lors de la phase de conception des valeurs en adéquation avec le mode de fabrication. Le comportement mécanique des nids d'abeilles Nomex® constituant l'âme de ces sandwichs est généralement validé à l'aide d'essais mécaniques ou de simulations éléments finis. Les bases théoriques du logiciel NidaCore développé au Laboratoire pour ce type d'étude sont présentées ci-après.

Dans la présente étude, la simulation par éléments finis des propriétés mécaniques élastiques homogénéisées d'âmes nids d'abeilles en papier Nomex® est menée à bien. Les contraintes ultimes de ces âmes sont déterminées en évaluant les charges critiques de flambements. L'influence des caractéristiques mécaniques en fonction de la hauteur de l'âme doit également être validée car les voiliers présentent des âmes de différentes épaisseurs. Les formes classiques hexagonales ainsi que leurs versions rectangulaires sur-expansées sont plus particulièrement étudiées. L'étude des âmes en Nomex® repose sur l'utilisation de la théorie de l'homogénéisation des milieux périodiques (Suquet, 1982, Debordes, 1982, Lene, 1984, Forest, 2006) et sur la détermination des modes de flambements de ces structures à parois fortement élancées. Le Volume Elémentaire Représentatif de chaque type de nids d'abeilles est mis en œuvre dans le code éléments finis Cast3MCEA (Verpeaux et al., 1988) afin de déterminer ces propriétés mécaniques homogénéisées. Toute la procédure d'homogénéisation, regroupée sous le nom de NidaCore est écrite en langage Gibiane propre à ce code. Afin de montrer la pertinence de l'approche développée dans cette étude, les contraintes ultimes de cisaillement déterminées à partir d'une étude de flambement sont comparées aux valeurs expérimentales issues des travaux du fabricant Euro-Composite. La présentation d'un des cas de chargement classiquement utilisé pour la validation des catamarans fait l'objet de la dernière partie. L'approche numérique multi-échelle que nous utilisons lors de l'étude des voiliers permet de faire des choix stratégiques de dimensionnement.

\section{Homogéneisation des milieux periodiques}

Lors des simulations Eléments Finis de validation de solution de conception ou de réparation de voiliers, l'ingénieur est souvent confronté à des matériaux ou à des structures fortement hétérogènes. Toutes les hétérogénéités ne peuvent pas être considérées individuellement et une procédure d'homogénéisation doit alors être menée afin de déterminer les caractéristiques mécaniques équivalentes.

L'homogénéisation consiste à substituer un milieu fortement hétérogène par un milieu fictif homogène que l'on souhaite équivalent dans une gamme de chargements la plus large possible. Le milieu homogène équivalent se comporte alors en moyenne comme le milieu hétérogène à condition de mesurer les propriétés mécaniques sur une échelle grande devant la taille des hétérogénéités. Les nids d'abeilles constituant l'âme des panneaux sandwichs sont des structures hétérogènes périodiques qui 
continuent de faire l'objet de nombreuses études. Les premiers travaux de Kelsey sur la modélisation des âmes ont porté sur la détermination des caractéristiques mécaniques des modules de cisaillement hors plan (Kelsey et al., 1958). Ces propriétés mécaniques sont déterminantes dans le comportement d'une structure sandwich. La caractérisation du comportement plan pour des formes hexagonales a ensuite été menée par (Gibson et al., 1982, Gibson et Ashby, 1988, Shang et Ashby, 1992) afin d'affiner les modèles analytiques. Enfin des approches Eléments Finis ont récemment été menées par Grediac afin de déterminer les bornes des modules de cisaillement hors plans d'âmes hexagonales (Grediac, 1993). Récemment, des techniques d'homogénéisation associées à des hypothèses cinématiques conduisant à des bornes de Voigt ont été mises en œuvre par (Hohe et Becker, 2001) sur des âmes alvéolaires. La théorie de l'homogénéisation des milieux périodiques permet de passer des grandeurs mécaniques des constituants élémentaires à des grandeurs mécaniques globales. Notre étude, fondée sur cette théorie, est générale et la résolution est menée dans le cadre des Eléments Finis. Les 21 coefficients du milieu homogénéisé anisotrope tridimensionnel sont déterminés à partir de la géométrie de l'âme et de ses propriétés mécaniques élémentaires. Notre approche est illustrée par des simulations sur des âmes de forme hexagonale classique ainsi que sur leurs versions rectangulaires sur-expansées. Lors de la phase de conception ou de réparation des voiliers océaniques, les propriétés mécaniques des âmes sont une des données essentielles pour le calcul de l'intégrité du voilier. La caractérisation mécanique des peaux de carbone utilisées sur les sandwichs doit également être validée afin de révéler les éventuelles faiblesses en compression ou cisaillement (Gornet et al., 2000). La mise en œuvre de cette théorie au sein du code de calculs Eléments Finis Cast3M développé par le CEA a conduit à la réalisation d'un programme spécifique dédié à la caractérisation mécanique des âmes périodiques.

\subsection{Volume Elémentaire Représentatif}

Toutes les théories d'homogénéisation nécessitent le choix d'un Volume Elémentaire Représentatif (VER). Les volumes élémentaires représentatifs retenus pour l'homogénéisation des nids d'abeilles à section hexagonale et rectangulaire surexpansé sont représentés sur la figure 2. Tout volume plus grand peut se déduire par des translations successives du VER. Il convient de noter cependant que le mode de dégradation peut dans certains cas être défini par un VER de dimension supérieure. En effet, les longueurs caractéristiques associées aux mécanismes de déformation et dégradation dans le cas de comportements non-linéaires peuvent parfois englober plusieurs cellules de VER. Dans le cas général d'un matériau de constitution irrégulière, le VER devra inclure un grand nombre d'hétérogénéités afin d'être effectivement représentatif du milieu considéré. Dans le cas des deux VER représentés sur la figure 2 , la base $\vec{x}, \vec{y}, \vec{z}$ correspond à la base d'orthotropie du matériau homogénéisé. 


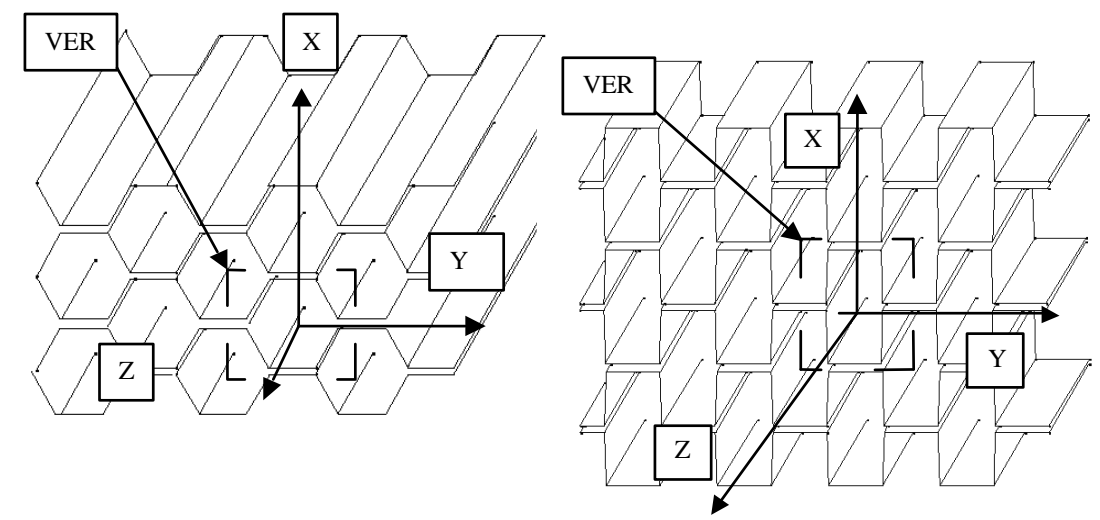

Figure 2. Maillage coque du catamaran code B1, avec son chargement dans la houle

\subsection{Localisation et méthodes d'homogénéisation}

Le processus mathématique qui permet de passer de grandeurs globales définies sur le VER aux grandeurs locales est appelé processus de localisation. En notant $Y$ le VER et $|Y|$ la mesure de son volume, la moyenne spatiale d'un champ de déformation ou de contrainte est donnée par l'équation (1).

$$
\left\langle X_{i j}\right\rangle=\frac{1}{|Y|} \int_{Y} X_{i j}(y) d y
$$

Les contraintes et les déformations moyennes sont notées respectivement : $\Sigma_{i j}=\left\langle\sigma_{i j}\right\rangle$ et $E_{i j}=\left\langle\varepsilon_{i j}\right\rangle$. On peut également définir les contraintes locales $\sigma_{i j}$ et les déformations locales $\varepsilon_{i j}$ à partir de ces grandeurs moyennes $\Sigma$ et $\mathrm{E}$. Lors du processus d'homogénéisation, les grandeurs microscopiques sont reliées aux grandeurs macroscopiques via les tenseurs d'ordre quatre de localisation $A$ et $B$. Les méthodes classiques d'homogénéisation reposent sur le choix des tenseurs de localisation reliant les grandeurs microscopiques aux grandeurs macroscopiques (2) :

$$
\sigma(y)=A(y): \Sigma \varepsilon(y)=B(y): \mathrm{E}
$$

Il convient de rappeler que si l'on fait l'hypothèse que les tenseurs de localisation $A$ ou $B$ sont des tenseurs identités la méthode d'homogénéisation conduit respectivement aux bornes classiques de Voigt et Reuss. 
Une homogénéisation à contraintes moyennes imposées avec l'hypothèse $B=I$ permet d'obtenir une souplesse du VER qui est la moyenne des souplesses locales. Cette méthode correspond à la méthode de Reuss $S_{\operatorname{Re} u s s}^{V E R}=\langle S\rangle$.

Une homogénéisation à déformations moyennes imposées avec l'hypothèse $A=I$ permet d'obtenir la rigidité du VER qui est la moyenne des rigidités locales. Cette hypothèse correspond à la méthode de Voigt $C_{\mathrm{Re} u s s}^{V E R}=\langle C\rangle$. Elle correspond à un état uniforme des déformations dans le VER.

Les hypothèses de Voigt et Reuss ne sont pas applicables simultanément sur un VER hétérogène. En conséquence $C_{\mathrm{Re} u s s}^{V E R}$ et $S_{\mathrm{Re} u s s}^{V E R}$ ne sont pas l'inverse l'une de l'autre. Une approche plus fine peut être menée en résolvant le problème d'élasticité posé sur le VER. Les conditions aux limites sur le bord du VER peuvent être les contraintes moyennes $\Sigma$ ou les déformations moyennes E. Dans la suite, la méthode des modules effectifs fondée sur la donnée des déformations moyennes $\mathrm{E}$ est appliquée aux cellules de nids d'abeilles. Cette méthode nous permet de déterminer le comportement homogénéisé élastique anisotrope de ces âmes. Il convient de noter que dans le cas de milieux périodiques, l'homogénéisation s'appuyant sur une approche à contrainte ou à déformation macroscopiques imposées conduit à un comportement équivalent qui découle du principe de HillMandel.

\subsection{Méthode des modules effectifs}

Cette méthode se fonde sur la donnée du champ de déformation moyenne $\mathrm{E}$ et conduit à déterminer l'opérateur de complaisance $C^{V E R}$ du VER à partir de conditions limites en déplacements imposés sur son bord $\partial Y=\partial Y_{21} \cup \partial Y_{22}$. La méthode conduit à un comportement homogénéisé avec le tenseur de localisation $A$ différent de l'identité. Le processus d'homogénéisation retenu est basé sur la résolution de 6 problèmes d'élasticité linéaire élémentaires (3-6). Ces six problèmes d'élasticité linéaire à résoudre correspondent à des conditions limites (6) telles que $E_{k h}=1$ et $E_{i j}=0$ si $(i, j) \neq(k, h)$. La détermination de la rigidité de l'âme nécessite quant à elle 21 combinaisons linéaires des six problèmes élémentaires. Les problèmes élémentaires à résoudre sont présentés ci-après :

$$
\begin{aligned}
& \operatorname{div}(\sigma(y))=0 \quad \sigma(y)=C(y) \varepsilon(u(y)) \quad \text { dans } Y \\
& \varepsilon(u)=\operatorname{grad}_{s}(u(y)) \\
& \sigma(y) \vec{n}_{e x t}=\overrightarrow{0} \quad \text { sur } \partial Y_{21} \\
& \vec{u}(y)=\mathrm{E} \vec{y}+\vec{v}_{p e r} \quad \text { sur } \partial Y_{22}
\end{aligned}
$$

Le champ de déplacement $\vec{v}_{p e r}$ est périodique et prend naturellement des valeurs égales sur les faces opposées du VER. La linéarité du problème traité permet de décomposer la solution en déformation $\mathrm{du}$ problème sous la forme 
$\varepsilon(y)=e^{k h}(y) E_{k h}$ où $e^{k h}(y)$ représente la solution du problème pour un cas d'un chargement élémentaire $E_{k h}$. En conséquence, le tenseur de localisation est construit à partir de la solution du problème posé sur le VER $A_{i j}{ }^{k h}=e_{i j}^{k h}$. Le tenseur des raideurs homogénéisé $C^{V E R}$ est alors donné par la formule suivante :

$$
C^{V E R}=\langle C(y): A(y)\rangle
$$

En pratique on utilise l'approche énergétique de Hill-Mandel et la notation vectorielle pour calculer les composantes de la rigidité homogénéisée équivalente $C^{V E R}$.

\subsection{Principe de macro-homogénéité de Hill-Mandel}

Les caractéristiques mécaniques homogénéisées du VER peuvent être déterminées simplement par l'approche énergétique qui est fondée sur le théorème de Hill-Mandel. Le théorème impose que l'énergie du VER est la moyenne de l'énergie de ces constituants (7) avec :

$$
\begin{aligned}
& \langle\sigma \varepsilon\rangle=\Sigma: E \\
& \Sigma=C^{V E R} E \\
& E=S^{V E R} \Sigma
\end{aligned}
$$

Il convient de noter que cette condition est vérifiée par l'ensemble des méthodes d'homogénéisation évoquées. Dans le cas des milieux périodiques, l'approche en contrainte et l'approche en déformation sont équivalentes [4].

$$
\frac{1}{|Y|} \int_{Y} \sigma: \varepsilon d y=E:\left(C^{V E R} E\right)
$$

L'approche précédente définit le comportement homogénéisé sous forme d'une loi élastique linéaire anisotrope que l'on détermine en résolvant les six problèmes élémentaires posés sur le VER. La loi de comportement inverse du matériau homogène équivalent est déterminée à partir de la relation $S^{V E R}=C^{V E R^{-1}}$. La mise en ouvre dans le code éléments finis Cast3M-CEA de la méthode d'homogénéisation précédente est réalisée à l'aide du langage de commandes Gibiane (Verpeaux et al. 1988). Le centre du repère est au centre du VER et les conditions de périodicité sont appliquées aux faces en vis-à-vis à l'aide de relations linéaires. Les bords $\partial Y_{22}$ sont les bords 1, 2, 3, 4, 5 et 6 définis sur la figure 3. Le cas particulier des cellules sur-expansées correspond à un angle $\alpha$ de $90^{\circ}$. Les conditions de chargement à déplacements imposés $\vec{u}(y)=\mathrm{E} \vec{y}+\vec{v}_{p e r}$ sur les bords $\partial Y_{22}$ sont traitées en remplaçant le champ $\vec{v}_{p e r}$ par des relations linéaires équivalentes. Ces relations traduisent la périodicité du VER. 


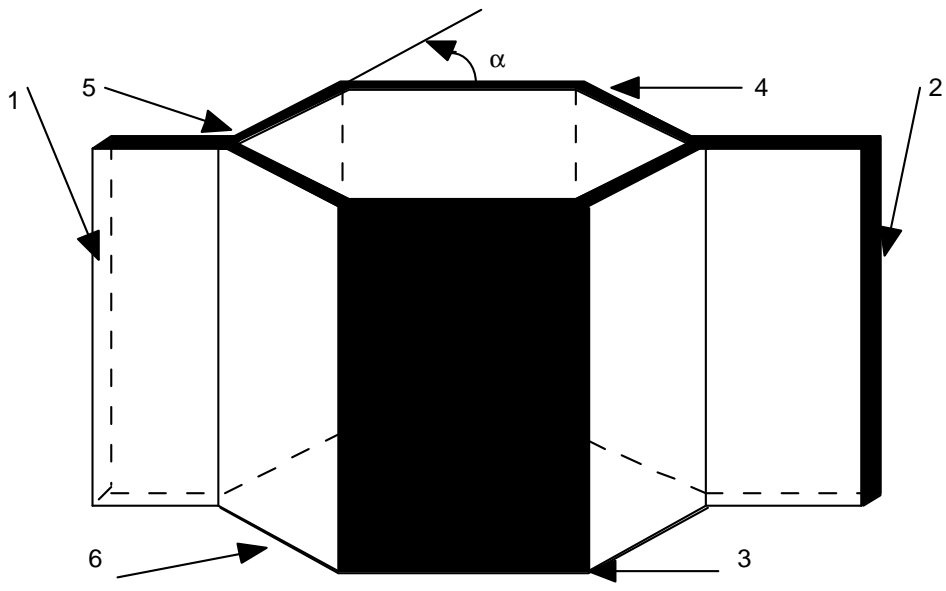

Figure 3. Cellules de nids d'abeilles hexagonales

\subsection{Calcul de la rigidité et de la souplesse}

Les coefficients du matériau homogénéisé sont déterminés à partir du théorème de Hill-Mandel en écrivant l'énergie à partir des expressions sous forme matricielle. Le tenseur des contraintes et celui des déformations sont des tenseurs symétriques d'ordre deux et le comportement élastique est représenté par un tenseur d'ordre quatre. Dans le cas des matériaux anisotropes, on utilise classiquement une notation vectorielle pour représenter leur comportement. La notation retenue dans cette étude diffère des notations classiques car elle est symétrisée et permet de simplifier les expressions des composantes de la rigidité et de la souplesse lors des opérations de changements de base (Gornet, 2001, Bornert et al., 2001). En notation vectorielle, les composantes des contraintes et des déformations dans le base notée $\vec{N}_{1}, \vec{N}_{2}, \vec{N}_{3}$ sont rangées dans deux vecteurs notés respectivement $\hat{\sigma}$ et $\hat{\varepsilon}$ :

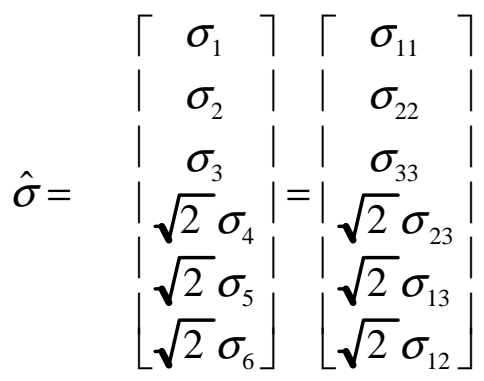$$
\hat{\mathcal{\varepsilon}}=\left|\begin{array}{c|c|c}
\varepsilon_{1} & \lceil & \varepsilon_{11} \\
\varepsilon_{2} & \mid & \varepsilon_{22} \\
\varepsilon_{3} & \mid & \varepsilon_{33} \\
\sqrt{2} \varepsilon_{4} \\
\sqrt{2} \varepsilon_{5} \\
\sqrt{2} & \varepsilon_{6}
\end{array}\right|=\left|\begin{array}{cc}
\sqrt{2} \varepsilon_{23} \\
\sqrt{2} \varepsilon_{13} \\
\sqrt{2} & \varepsilon_{12}
\end{array}\right|
$$ 
La densité d'énergie interne de déformation est retrouvée en fonction des représentations vectorielles des contraintes et des déformations $: \operatorname{Tr}[\sigma \varepsilon]=\hat{\sigma}^{T} \hat{\mathcal{E}}$. La loi de comportement du nid d'abeille homogénéisé s'écrit sous la forme suivante dans la base $\vec{N}_{1}, \vec{N}_{2}, \vec{N}_{3}: \hat{\sigma}=\hat{C} \hat{\varepsilon}, \quad \hat{\sigma}_{I}=\hat{C}_{I J} \hat{\varepsilon}_{J}$ avec $\hat{C}_{I J}=\hat{C}_{J I}$.

La relation inverse s'écrit : $\hat{\varepsilon}=\hat{S} \hat{\sigma}$ avec $\hat{S}=\hat{C}^{-1}, \varepsilon_{I}=\hat{S}_{I J} \sigma_{J}$. Lorsque l'âme est quelconque et ne présente pas de symétrie élastique, elle est caractérisée par 21 constantes élastiques indépendantes $\hat{S}_{I J}=\hat{C}_{I J}^{-1}$. La géométrie des âmes considérées dans cette étude comporte trois plans de symétrie perpendiculaires ce qui conduit naturellement à un comportement homogénéisé orthotrope. Le comportement est défini par la base d'orthotropie $\vec{x}, \vec{y}, \vec{z}$ (figure 2) et les 9 coefficients d'élasticité indépendants dans cette base. La condition de Hill-mandel est écrite sous forme matricielle afin de simplifier la mise en œuvre Eléments Finis (12-13) :

$$
\begin{aligned}
& |Y|^{V E R} \hat{\sigma}^{T} \hat{\varepsilon}=\int_{Y} \operatorname{Tr}[\sigma \varepsilon] \\
& \hat{\mathcal{\varepsilon}}_{I}^{T} \hat{C}_{I J}^{V E R} \hat{\mathcal{\varepsilon}}_{J}=\frac{1}{|Y|^{V E R}} \int_{Y} \operatorname{Tr}[\sigma \varepsilon] d Y
\end{aligned}
$$

Les 21 combinaisons linéaires des six problèmes d'élasticité élémentaires posés sur le VER permettent de déterminer entièrement la matrice de rigidité $\hat{C}_{I J}$.

Les équations sont développées ci après. Il n'y a pas sommation sur les indices dans les formules suivantes :

$$
\begin{gathered}
\varepsilon_{I} \hat{C}_{I I}^{V E R} \varepsilon_{I}=\frac{1}{|Y|^{V E R}} \int_{Y} \operatorname{Tr}[\sigma \varepsilon] d Y \quad \text { pour } I \in[1,3] \\
\sqrt{2} \varepsilon_{I} \hat{C}_{I I}^{V E R} \sqrt{2} \varepsilon_{I}=\frac{1}{|Y|^{V E R}} \int_{Y} \operatorname{Tr}[\sigma \varepsilon] d Y \quad \text { pour } I \in[4,6] \\
\varepsilon_{I} C_{I I}^{V E R} \varepsilon_{I}+2 \varepsilon_{I} C_{I J}^{V E R} \varepsilon_{J}+\varepsilon_{J} C_{J J}^{V E R} \varepsilon_{J}=\frac{1}{|Y|^{V E R}} \int_{Y} \operatorname{Tr}[\sigma \varepsilon] d Y \\
\operatorname{pour}(I, J) \in[1,3]^{2}, \text { avec }(I \neq J) \\
\left.\varepsilon_{I} C_{I I}^{V E R} \varepsilon_{I}+2 \varepsilon_{I} C_{I J}^{V E R} \sqrt{2} \varepsilon_{J}+\sqrt{2} \varepsilon_{J} C_{J J}^{V E R} \sqrt{2} \varepsilon_{J}=\frac{1}{|Y|^{V E R}} \int_{Y} \operatorname{Tr}[\sigma \varepsilon] d Y\right) \\
\sqrt{2} \varepsilon_{I} C_{I I}^{V E R} \sqrt{2} \varepsilon_{I}+2 \sqrt{2} \varepsilon_{I} C_{I J}^{V E R} \sqrt{2} \varepsilon_{J}+\sqrt{2} \varepsilon_{J} C_{J J}^{V E R} \sqrt{2} \varepsilon_{J}=\frac{1}{|Y|^{V E R}} \int_{Y} \operatorname{Tr}[\sigma \varepsilon] d Y \\
\operatorname{pour}(I, J) \in[4,6]^{2}, \text { avec }(I \neq J)
\end{gathered}
$$


Les équations (14-18) permettent de déterminer facilement la représentation du tenseur des rigidités $\hat{C}_{I J}$ dans la base retenue. Les modules d'Young, coefficient de Poisson ou de couplage sont définis classiquement à partir de la souplesse $\hat{S}_{I J}=\hat{C}_{I J}^{-1}$. Le comportement hors de la base d'orthotropie fait apparaittre des couplages avec le comportement de cisaillement. Un des avantages de l'approche vectorielle symétrisée introduite dans cette étude est d'obtenir des relations de changement base analytiques identiques sur la rigidité et la souplesse contrairement à la notation classiquement admise (Herakovich, 1998).

\section{Propriétés mécaniques des nids d'abeilles}

Les nids d'abeilles réalisés en papier Nomex® sont disponibles chez les fabricants pour différentes densités. Dans cette étude, les caractéristiques mécaniques du papier Nomex® sont déterminées de manière inverse par recalage des modules de cisaillement hors plan de l'âme homogénéisée. Les exemples qui illustrent cette étude sont déterminés à partir du catalogue «Euro-Composite». Il convient de noter qu'un produit peut avoir pour une même dimension géométrique, différentes densités, car la quantité de résine déposée peut varier afin de créer une âme plus résistante. Les catalogues des fabricants donnent uniquement les propriétés mécaniques les plus importantes vis-à-vis du dimensionnement. Elles sont définies généralement à partir d'essais réalisés pour des hauteurs de nid d'abeilles de $12 \mathrm{~mm}$. Les modules de cisaillement hors plan $\mathrm{G}_{13}$ et $\mathrm{G}_{23}$ et les contraintes de cisaillement de rupture associées $\sigma_{13}$ et $\sigma_{23}$ ainsi que la contrainte d'écrasement $\sigma_{33}$ sont les seules données fournies classiquement par les constructeurs. Ces seules données sont évidemment insuffisantes pour réaliser des études Eléments Finis tridimensionnelles. L'homogénéisation des milieux périodiques décrite ci-dessus permet alors de déterminer l'ensemble des caractéristiques mécaniques équivalentes d'un matériau nids d'abeilles.

\subsection{Simulations éléments finis}

Les simulations des propriétés mécaniques homogénéisées sont déterminées à partir des VER des nids d'abeilles présentés sur les figures 4 et 5 . Ils possèdent des hauteurs de $12 \mathrm{~mm}$ conformément au matériau utilisé par le fabriquant pour réaliser les tests mécaniques. Le modèle éléments finis d'un VER comporte 41556 nœuds, soit 6156 éléments hexaèdres à 20 nœuds isoparamétriques à interpolation quadratique de la famille de Serendip. L'intégration numérique est réalisée à l'aide de 27 points de Gauss. Le maillage d'un VER comporte un seul élément dans l'épaisseur élémentaire de la paroi et deux dans les parties à épaisseur double (figures 4 et 5). Des études de convergence ont été réalisées pour différentes discrétisations des VER et les maillages retenus sont optimaux vis-à-vis du temps CPU et de la convergence EF. Les simulations éléments finis conduisant aux propriétés mécaniques homogénéisées sont effectuées sous l'hypothèse des petits 
déplacements et des petites déformations. L'observation des modes de rupture des nids d'abeilles (Gibson et Ashby, 1988, Aminanda et al., 2003, Gornet et al., 2006) nous conduit à déterminer les contraintes de rupture de ces matériaux en utilisant la théorie du flambement d'Euler. Le langage de programmation du code Cast3M nous permet d'appeler au sein de nos propres développements le module de flambement qui correspond à la recherche de valeurs propres et de vecteurs propres. Les déformations homogénéisées critiques déterminées par le flambement sont introduites dans la loi de comportement homogénéisée afin de déterminer les contraintes ultimes.

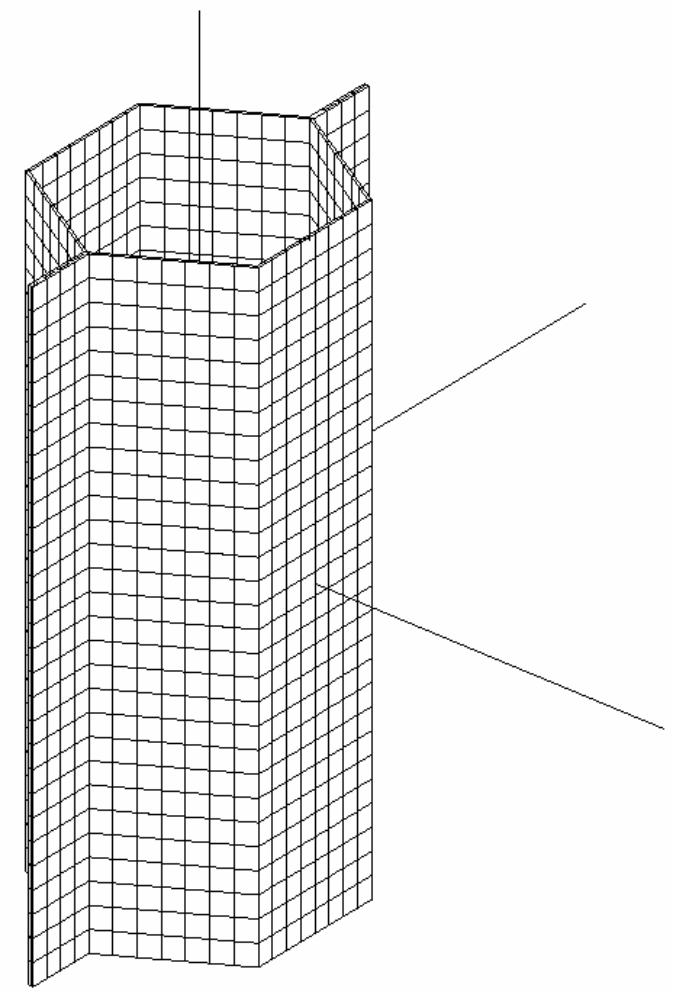

Figure 4. Maillage d'un VER de nids d'abeilles hexagonales 


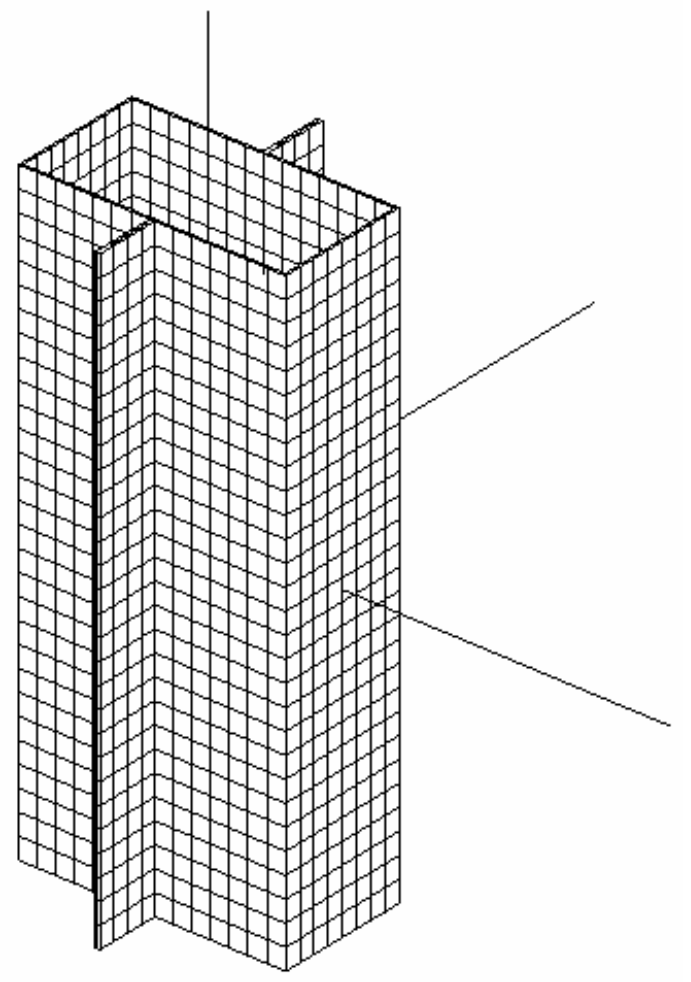

Figure 5. Maillage d'un VER de nids d'abeilles sur expansés

\subsection{Caractéristiques mécaniques}

Le calibrage des caractéristiques mécaniques du VER représentant le nid d'abeilles retenu est réalisé en supposant que les parois sont élastiques isotropes. En conséquence, le module d'Young du Nomex® et le coefficient de Poisson sont déterminés afin d'obtenir les modules de cisaillement hors plan du matériau orthotrope équivalent en adéquation avec le catalogue du fournisseur. Il convient de noter que ces modules sont déterminés expérimentalement à partir d'essais réalisés sur des éprouvettes de cisaillement. La démarche d'étalonnage du modèle Eléments Finis est nécessaire pour chaque densité d'âme pour une même géométrie. Le catalogue précise pour chaque géométrie les valeurs minimales (Min) et typiques (Typ) obtenues pendant les essais. En conséquence, deux calibrages sont effectués afin de déterminer les caractéristiques homogénéisées nominales et minimales.

Il convient de noter que les 21 coefficients du milieu homogène équivalent sont calculés. Dans le cas des nids d'abeilles à cellules hexagonales ou 
rectangulaires, les symétries conduisent à un comportement mécanique homogénéisé orthotrope. La représentation du VER dans la base d'orthotropie permet de déterminer uniquement les 9 coefficients d'élasticité linéaire du milieu homogène équivalent. Le modèle Eléments Finis du VER fournit après simulations une approximation de qualité des propriétés mécaniques de l'ensemble du matériau équivalent.

La dénomination ECA-R 4,8-29 (51) représente une âme rectangulaire de cercle inscrit de $4,8 \mathrm{~mm}$ de densité de $29 \mathrm{Kg} / \mathrm{m}^{3}$ et d'une épaisseur de clinquant de $51 \mu \mathrm{m}$. La dénomination ECA 4,8-29 (51) est équivalente pour une âme de section classique hexagonale. Les propriétés mécaniques homogénéisées des nids d'abeilles de géométrie rectangulaire sont présentées dans les tableaux I et II et les géométries hexagonales sont fournies dans les tableaux III à IV. Les propriétés mécaniques de cisaillement hors plan sont présentées sur les figures 6 et 7 pour les matériaux ECA 4,8 et ECA-R 4,8. L'évolution des propriétés mécaniques en fonction de la masse volumique est présentée sur la figure 8 pour le matériau hexagonal et sur la figure 9 pour sa version sur-expansée.

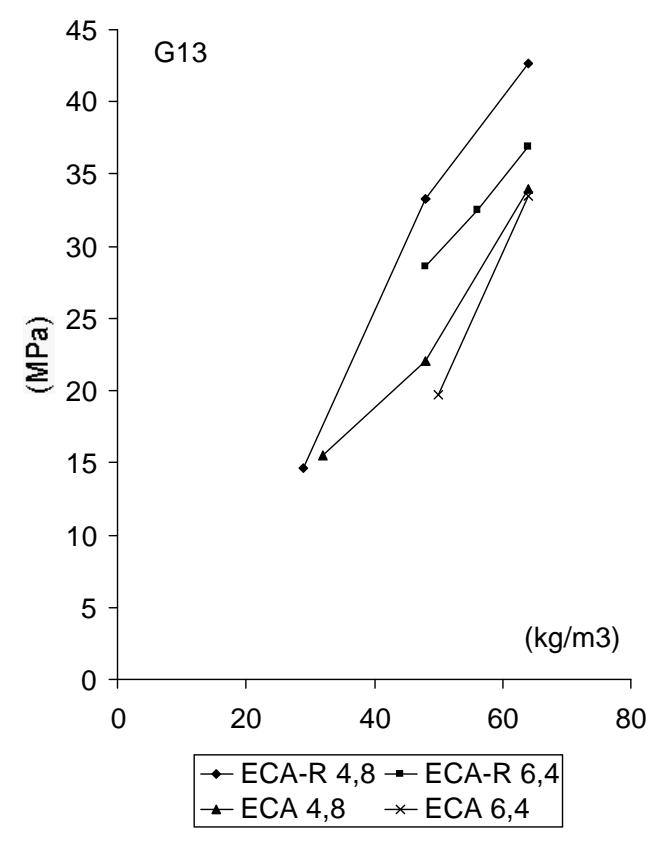

Figure 6. Maillage d'un VER de nids d'abeilles sur expansés 


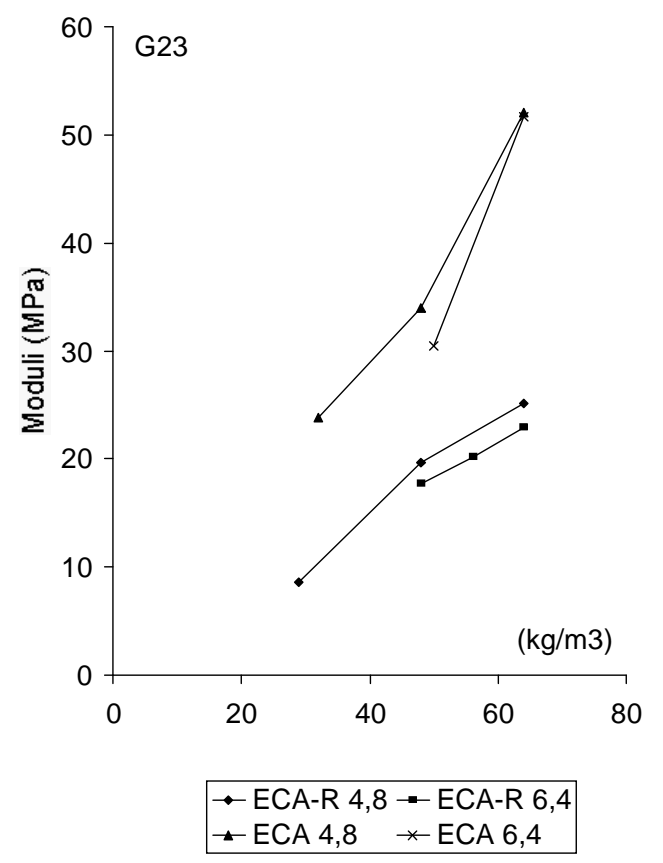

Figure 7. Evolution du module de cisaillement $G_{23}$ pour deux matériaux à cellules hexagonales et sur-expansées 


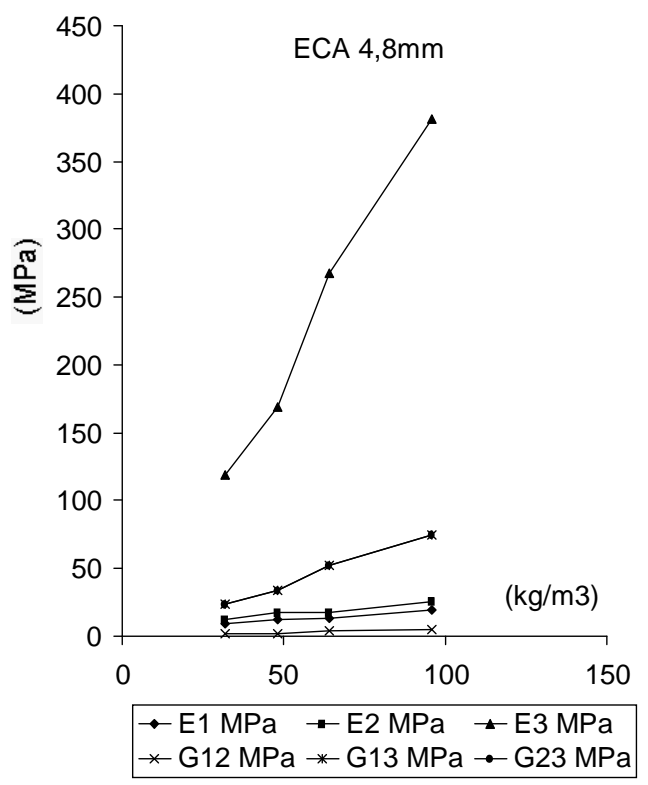

Figure 8. Evolution des propriétés mécaniques du ECA 4,8 en fonction de sa masse volumique 


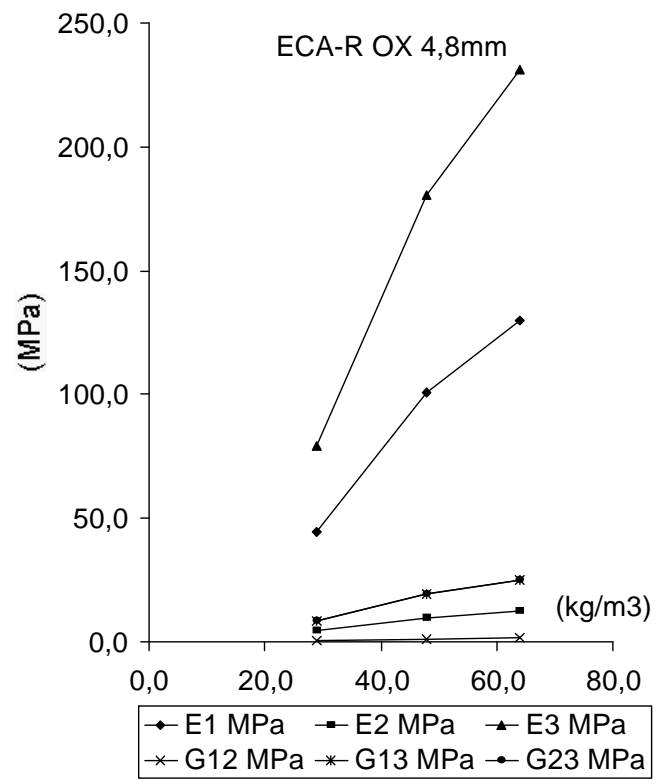

Figure 9. Evolution des propriétés mécaniques du ECA- $R$ 4,8 en fonction de sa masse volumique

Dans cette étude, les peaux ne sont pas modélisées et les conditions limites appliquées au VER sont équivalentes à des peaux infiniment rigides. Il convient de noter que les propriétés mécaniques de l'âme dépendent théoriquement de la rigidité des peaux. En réalité, les peaux sont quasiment rigides vis-à-vis de l'âme ce qui nous permet d'avoir des résultats satisfaisants. Les échantillonnages des voiliers de compétition sont déterminés avec des marges de sécurité extrêmement faibles afin d'alléger au maximum la structure et une bonne connaissance des matériaux les constituants demeure une préoccupation constante. En conséquence les peaux des sandwichs ont généralement des épaisseurs très faibles devant l'épaisseur de l'âme et les phénomènes de flambement restent un facteur de risque très important pour ce type de structure. 


\begin{tabular}{|l|c|c|c|c|c|c|}
\hline $\begin{array}{c}\text { ECA-R } \\
\text { MPa }\end{array}$ & $\begin{array}{c}\text { Min } \\
4,8-29 \\
(51)\end{array}$ & $\begin{array}{c}\text { Typ } \\
4,8-29 \\
(51)\end{array}$ & $\begin{array}{c}\text { Min } \\
4,8-48 \\
(51)\end{array}$ & $\begin{array}{c}\text { Typ } \\
4,8-48 \\
(51)\end{array}$ & $\begin{array}{c}\text { Min } \\
4,8-64 \\
(51)\end{array}$ & $\begin{array}{c}\text { Typ } \\
4,8-64- \\
(51)\end{array}$ \\
\hline \hline v12 & \multicolumn{2}{|c|}{0,2606} & \multicolumn{2}{|c|}{0,2606} & \multicolumn{2}{c|}{0,2606} \\
v13 & \multicolumn{2}{|c|}{0,2240} & \multicolumn{2}{c|}{0,2240} & \multicolumn{2}{c|}{0,2240} \\
v23 & \multicolumn{2}{|c|}{0,0219} & \multicolumn{2}{c|}{0,0219} & \multicolumn{2}{c|}{0,0219} \\
\hline E1 & 44,5 & 72,5 & 101,1 & 128,7 & 129,6 & 152,0 \\
E2 & 4,4 & 7,1 & 9,9 & 12,6 & 12,7 & 14,9 \\
E3 & 79,4 & 129,5 & 180,5 & 229,8 & 231,4 & 271,5 \\
\hline \hline G12 & 0,5 & 0,9 & 1,2 & 1,6 & 1,6 & 1,9 \\
G13 W & 14,6 & 23,9 & 33,3 & 42,4 & 42,7 & 50,1 \\
G23 L & 8,6 & 14,1 & 19,6 & 25,0 & 25,1 & 29,5 \\
\hline
\end{tabular}

Table 1. Comparaisons entre les caractéristiques éléments finis (EF) et constructeur (EC). Modules de cisaillement minimum (Min) et typiques (Typ) des nids d'abeilles ECA-R 4,8 de densité 29, 48 et $64 \mathrm{Kg} / \mathrm{m}^{3}$.

\begin{tabular}{|c|c|c|c|c|c|c|c|c|c|c|c|c|}
\hline \multirow{3}{*}{$\begin{array}{c}\text { ECA -R } \\
\mathrm{MPa} \\
\end{array}$} & \multicolumn{4}{|c|}{$4,8-29(51)$} & \multicolumn{4}{|c|}{ 4,8-48 (51) } & \multicolumn{4}{|c|}{$4,8-64(51)$} \\
\hline & \multicolumn{2}{|c|}{ Min } & \multicolumn{2}{|c|}{ Typ } & \multicolumn{2}{|c|}{ Min } & \multicolumn{2}{|c|}{ Typ } & \multicolumn{2}{|c|}{ Min } & \multicolumn{2}{|c|}{ Tyр } \\
\hline & EF & EC & EF & EC & EF & EC & EF & EC & EF & EC & EF & EC \\
\hline $\begin{array}{l}\text { G13 W } \\
\text { G23 L }\end{array}$ & \begin{tabular}{|c|}
14,6 \\
8,6
\end{tabular} & $\begin{array}{c}14 \\
9\end{array}$ & \begin{tabular}{|l}
23,9 \\
14,1
\end{tabular} & $\begin{array}{l}24 \\
14\end{array}$ & \begin{tabular}{|l}
33,3 \\
19,6
\end{tabular} & $\begin{array}{l}36 \\
18\end{array}$ & $\begin{array}{l}42,4 \\
25,0\end{array}$ & $\begin{array}{l}44 \\
24\end{array}$ & $\begin{array}{l}42,7 \\
25,1\end{array}$ & $\begin{array}{l}48 \\
22\end{array}$ & \begin{tabular}{|l||}
50,1 \\
29,5
\end{tabular} & $\begin{array}{l}56 \\
26\end{array}$ \\
\hline
\end{tabular}

Table 2. Comparaisons entre les caractéristiques éléments finis (EF) et constructeur (EC). Modules de cisaillement minimum (Min) et typiques (Typ) des nids d'abeilles ECA-R 6,4 de densité 48, 56 et $64 \mathrm{Kg} / \mathrm{m}^{3}$.

\begin{tabular}{|c|c|c|c|c|c|c|c|c|c|c|c|c|}
\hline \multirow{3}{*}{$\begin{array}{c}\text { ECA - R } \\
\mathrm{MPa} \\
\end{array}$} & \multicolumn{4}{|c|}{$6,4-48(51)$} & \multicolumn{4}{|c|}{$6,4-56(51)$} & \multicolumn{4}{|c|}{$6,4-64(51)$} \\
\hline & \multicolumn{2}{|c|}{ Min } & \multicolumn{2}{|c|}{ Typ } & \multicolumn{2}{|c|}{ Min } & \multicolumn{2}{|c|}{ Typ } & \multicolumn{2}{|c|}{ Min } & \multicolumn{2}{|c|}{ Typ } \\
\hline & EF & $\overline{E \text { EC }}$ & $\overline{E \overline{E F}}$ & $\overline{E C}$ & $\overline{E F}$ & EC & $\overline{E F}$ & $\overline{E \text { EC }}$ & $\overline{E \text { EF }}$ & $\overline{E \text { EC }}$ & EF & $\overline{E C}$ \\
\hline $\begin{array}{l}\text { G13 W } \\
\text { G23 L }\end{array}$ & $\begin{array}{l}28,6 \\
17,7\end{array}$ & $\begin{array}{l}33 \\
15\end{array}$ & \begin{tabular}{|l|}
38,7 \\
24,0
\end{tabular} & $\begin{array}{l}42 \\
22\end{array}$ & $\begin{array}{l}32,5 \\
20,2\end{array}$ & $\begin{array}{l}33 \\
18\end{array}$ & \begin{tabular}{|l|}
42,4 \\
26,3
\end{tabular} & $\begin{array}{l}42 \\
24\end{array}$ & \begin{tabular}{|l|}
36,9 \\
22,9
\end{tabular} & $\begin{array}{l}40 \\
21\end{array}$ & \begin{tabular}{|l|}
49,0 \\
30,4
\end{tabular} & $\begin{array}{l}56 \\
26\end{array}$ \\
\hline
\end{tabular}

Table 3. Comparaisons entre les caractéristiques éléments finis (EF) et constructeur (EC). Modules de cisaillement minimum (Min) et typiques (Typ) des nids d'abeilles ECA 4,8 de densité 32, 48 et $64 \mathrm{Kg} / \mathrm{m}^{3}$. 


\begin{tabular}{|c|c|c|c|c|c|c|c|c|c|c|c|c|}
\hline \multirow{3}{*}{$\begin{array}{l}\text { ECA } \\
\mathrm{MPa} \\
\end{array}$} & \multicolumn{4}{|c|}{$\begin{array}{c}4,8-32 \\
(51) \\
\end{array}$} & \multicolumn{4}{|c|}{$4,8-48(51)$} & \multicolumn{4}{|c|}{$4,8-64(76)$} \\
\hline & \multicolumn{2}{|c|}{ Min } & \multicolumn{2}{|c|}{ Typ } & \multicolumn{2}{|c|}{ Min } & \multicolumn{2}{|c|}{ Typ } & \multicolumn{2}{|c|}{ Min } & \multicolumn{2}{|c|}{ Typ } \\
\hline & 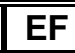 & EC & EF & EC & 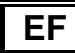 & EC & $\bar{E}$ EF & EC & $E F$ & EC & EF & EC \\
\hline 13 & $\begin{array}{l}5,5 \\
3,8\end{array}$ & $\begin{array}{l}16 \\
23\end{array}$ & $\begin{array}{l}1,1 \\
2,4\end{array}$ & $\begin{array}{l}22 \\
31\end{array}$ & $\begin{array}{l}22,1 \\
33,9\end{array}$ & $\begin{array}{l}22 \\
34\end{array}$ & $\begin{array}{l}27,0 \\
41,5\end{array}$ & $\begin{array}{l}28 \\
40\end{array}$ & $\begin{array}{l}33,9 \\
52,1\end{array}$ & $\begin{array}{l}34 \\
52\end{array}$ & $\begin{array}{l}43,8 \\
67,3\end{array}$ & \\
\hline
\end{tabular}

Table 4. Comparaisons entre les caractéristiques éléments finis $(E F)$ et constructeur (EC). Modules de cisaillement minimum (Min) et typiques (Typ) des nids d'abeilles ECA 4,8-96 et ECA 6,4 de densité, 50 et $64 \mathrm{Kg} / \mathrm{m}^{3}$.

\subsection{Contraintes à Rupture}

Expérimentalement, on constate que le flambement correspond à un accroissement très significatif de l'endommagement des âmes nids d'abeilles en Nomex®. Lors d'un essai mécanique de cisaillement ou de compression (Aminanda 2003, Gornet et al. 2006) hors plan, dès que la force atteint son maximum, une chute plus ou moins brutale de celle-ci est observée. Cette chute correspond à des plissements des parois. Ces matériaux sont connus pour avoir un comportement mécanique élastique linéaire fragile jusqu'à leur charge critique. En conséquence, nous considérerons que cette charge critique correspond à leur limite d'utilisation. Les déformations critiques sont déterminées pour l'ensemble des cas de chargements. L'étude des modes de flambement élastiques linéaires d'Euler (19) permet de déterminer les déformations critiques (20) :

$$
\left[K+\lambda^{2} K_{s}\right] \vec{X}_{\lambda}=\overrightarrow{0}
$$

Où $K$ est la raideur du VER, $K_{s}$ représente la raideur géométrique due à la précontrainte $s . \lambda^{2}$ est le coefficient multiplicateur du chargement. $\vec{X}_{\lambda}$ est le mode propre de flambement associé à $\lambda^{2}$. Les déformations critiques sont déterminées par la relation (20).

$$
E_{J}^{c}=\lambda^{2} E_{J}
$$

Dans le cas d'un matériau dont le comportement homogénéisé est orthotrope nous proposons de déterminer les six contraintes critiques élémentaires associées aux déformations critiques $E_{J}^{C}$ en appliquant la loi de comportement homogénéisée (21).

$$
\hat{\sigma}_{I}^{c}=\hat{C}_{I J} \hat{E}_{J}^{c}
$$

Les contraintes ultimes du matériau ECA-R 4,8 29 (51) sont présentées dans le tableau V. D'un point de vue physique, la couche de résine casse lors du premier 
plissement et la résistance de la cellule chute alors de façon très significative. Expérimentalement, le plateau sur la courbe représentant l'effort global en fonction du déplacement correspond à une densification du nid d'abeilles dû à l'écrasement des parois. D'un point de vue industriel, les zones écrasées du Nomex® conduisent à des flambements des peaux du sandwich qui dans certains cas peuvent entraîner la perte du navire. En conséquence, pour ces âmes dont le comportement élémentaire du papier enduit de résine est élastique fragile, des simulations éléments finis des modes de flambement du VER permettent de déterminer correctement les contraintes à rupture de l'âme homogénéisée. Ces contraintes à rupture du matériau orthotrope équivalent sont proches des résultats des essais fournis par le fabricant. Les contraintes ultimes peuvent être utilisées avec un critère de rupture de Hill, cependant cette démarche doit encore être validée expérimentalement. Ces premiers résultats sont très encourageants et montrent la pertinence de la modélisation retenue dans cette étude. L'étude du premier mode de flambement statique des VER fournit des résultats dignes de confiance (figure 10 ). Cependant, il convient de rappeler que de nombreux paramètres influent sur le flambement. Les plus connus sont le comportement du matériau, les défauts géométriques, les charges suiveuses ou encore, les conditions aux limites. Il est important de parfaitement maîtriser dans la simulation éléments finis, l'effet de chaque paramètre si l'on veut prédire de façon fiable le flambement qui conduit dans notre cas à la rupture du nid d'abeilles.
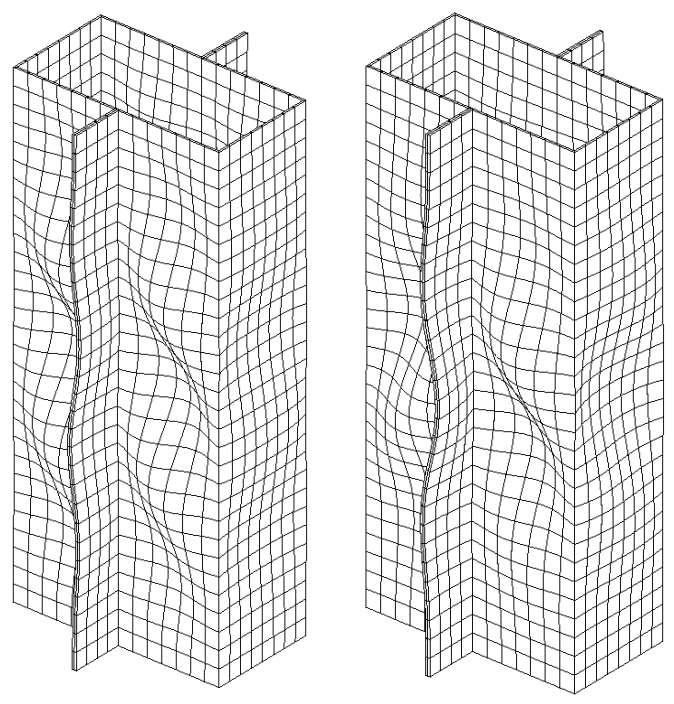

Figure 10. Déformée de flambement du VER sur-expansé sous l'action d'un chargement de cisaillement hors plan $E_{13}$. Déformée de flambement du VER surexpansé sous l'action d'un chargement de cisaillement hors plan $E_{23}$. 


\section{Etude d'un catamaran dans la houle}

Sur un voilier multicoque de 125 pieds, la structure est construite en matériaux composites sandwichs et elle subit des sollicitations de type flexion ou torsion. Ces voiliers sont constitués de sandwich composée d'âmes Nomex® et de peaux en carbone époxy. L'assemblage est réalisé par collage à l'aide de résine compatible avec les matériaux en présence. Les peaux sont généralement constituées de matériaux composites stratifiés en carbone époxy. Ces structures sandwich ont une grande rigidité en flexion et torsion. L'âme de la structure sandwich résiste principalement aux contraintes de cisaillement, de traction et de compression hors plan, les peaux inférieures et supérieures supportent quant à elles les efforts dans leur plan. Les peaux résistent principalement aux contraintes de traction et de compression générées par les moments de flexion. Elles doivent également résister à des flambements locaux qui peuvent être générés par des impacts hydrodynamiques. L'âme doit résister aux contraintes de cisaillement générées par le glissement des deux peaux sous l'action des chargements. Une rupture en cisaillement de l'âme générera un mouvement indépendant des peaux qui conduira à terme à des flambements des peaux et à leur destruction.

\subsection{Les efforts sur la structure}

La validation de la conception d'un maxi catamaran de 125 pieds repose sur une connaissance précise des sollicitations sur le voilier et des caractéristiques mécaniques des âmes et des matériaux composites carbone époxy le constituant. Les efforts extérieurs sur la structure du voilier sont dus au vent et aux forces hydrodynamiques.

Lorsque le voilier est posé sur l'eau, à l'arrêt il est soumis à l'équilibre de deux forces, celle de la pesanteur, dont la résultante est appliquée au centre de gravité, à laquelle s'oppose la force hydrostatique appliquée au centre de carène. La force propulsive d'origine vélique générera une force de résistance d'origine hydrodynamique sur les coques et appendices du voilier. L'ensemble mât-grémentsvoilure soumis aux effets du vent joue le rôle du moteur. Les coques assurent la flottabilité et sont soumises aux efforts hydrostatiques, hydrodynamiques et d'antidérive. Les bras de liaison qui solidarisent les deux coques sont une partie qui est tout particulièrement étudiée car c'est par ces éléments de liaison que transitent les efforts issus de la propulsion vers les coques en appuis sur la mer. Le dimensionnement précis de cette partie du voilier est capital pour sa durabilité mais également pour sa compétitivité. En effet, le rapport entre le poids et la puissance est un critère essentiel pour que les performances demeurent intéressantes pour toutes les conditions de vent et de mer qui seront rencontrées. Dans cette étude, les efforts d'origine vélique sont introduits sur le plan de pont. Ces charges concentrées ou réparties sont appliquées sur le pont. En réalité, les écoutes et les drisses sont accrochées sur le pont via des winches de manœuvre et renvoyée par des systèmes de 
poulies. L'action du vent sur le voilier se traduit par des efforts appliqué sur les coques ou sur les bras de liaison. Les efforts suivants doivent être pris en compte : efforts de traction dans l'étai, efforts de traction dans les haubans au vent, efforts de compression en pied de mât, efforts d'anti-dérive hydrostatique qui s'oppose aux efforts de dérive vélique.

L'oscillation permanente de la surface de la mer est communément appelée la houle. Elle est une conséquence de l'action du vent sur l'eau. L'échelle de BEAUFORT (Table 5) donne une relation empirique entre la vitesse du vent et la hauteur observable des vagues. L'oscillation de la surface libre se propage à une célérité qui dépend à la fois de la période de l'onde et du rapport d'onde à la profondeur de l'océan. La plus grande partie de l'énergie contenue dans la houle correspond à des périodes comprises entre 1 à 30 secondes. En conséquence, le voilier doit être dimensionné en tenant compte de sa vitesse d'avance dans la houle. Des analyses déterministes du voilier dans la houle sont présentées ci-après.

\begin{tabular}{|c|l|c|c|}
\hline Force Beaufort & \multicolumn{1}{|c|}{ Descriptif } & $\begin{array}{c}\text { Vitesse moyenne } \\
\text { du vent (Nouds) }\end{array}$ & $\begin{array}{c}\text { Hauteur significative } \\
\text { des vagues (m) }\end{array}$ \\
\hline 0 & Calme & $<1$ & 0 \\
1 & Très légère brise & 1 à 3 & 0,1 \\
2 & Légère brise & 4 à 6 & 0,3 \\
3 & Petite brise & 7 à 10 & 0,8 \\
4 & Jolie brise & 11 à 16 & 1,5 \\
5 & Bonne brise & 17 à 21 & 2,5 \\
6 & Vent frais & 22 à 27 & 3,5 \\
7 & Grand frais & 28 à 33 & 5 \\
8 & Coup de vent & 34 à 40 & 7 \\
9 & Fort coup de vent & 41 à 47 & 9 \\
10 & Tempête & 48 à 55 & 12 \\
11 & Violente tempête & 56 à 63 & 14 \\
12 & Ouragan & $>64$ & $>14$ \\
\hline
\end{tabular}

Table 5. Echelle de Beaufort. Interactions entre la vitesse du vent et la hauteur des vagues

\subsection{Description du modèle éléments finis}

Le modèle Eléments Finis du catamaran a été réalisé à partir des fichiers DAO (figure 1). Il est constitué principalement d'éléments finis coques multicouches. Le modèle est constitué de 57355 éléments coques. Ces éléments sont basés sur une extension au cas des stratifiés des théories développées par E. Reissner et R.D. Mindlin pour les matériaux homogènes isotropes. Le modèle comporte 288395 
degrés de liberté qui s'appuient sur 164837 nœuds. Il existe plus de 600 zones de stratification qui évoluent continuellement sur les coques et bras du voilier. Les flotteurs, les bras, la poutre avant, le longeron, la martingale et les dérives ont été retenus dans le modèle éléments finis. Les structures internes et les différentes ouvertures des cabines, trappe de visite et les puits de dérive sont également pris en compte. Les coques et les bras sont raidis par un ensemble complexe de cloisons de planchers et de lisses.

\subsection{Description du cas de charge "Houle Diagonale"}

Dans cette partie, le navire avance dans une houle orientée suivant sa diagonale. La longueur d'onde de la houle critique correspond à la diagonale du voilier. Dans le cas du «code B1», cette diagonale correspond à une houle de période 5,03s. L'amplitude de cette houle doit être compatible avec la vitesse d'avance du catamaran. Les pressions hydrodynamiques correspondant à cette configuration sont calculées à l'aide du logiciel Aquadyn développé à l'Ecole Centrale Nantes (Delhommeau, 1988). Les phases de la houle sont imposées sur la structure du voilier afin de valider le dimensionnement des structures (figure 11). Ce type de chargement proposé par l'Ecole centrale est à rapprocher des cas de chargements conventionnels présentés par Det Norske Veritas (1996). Le critère de Tsai-Wu (figure 12) est présenté sur l'ensemble de la structure lors d'une avancée du voilier dans la phase de houle la plus dimensionnant (figure 11). Le voilier présente un critère de Tsai relativement uniforme à l'exception de quelques zones fortement localisées. Ce type de simulation permet d'aider l'équipe de conception lors du choix de l'échantillonnage des structures. Un grand nombre d'autre cas de chargement sont évalués afin de créer une structure optimisée vis-à-vis de l'ensemble des modes de fonctionnement du voilier. 
Ames Nomex® : design d'un voilier multicoque

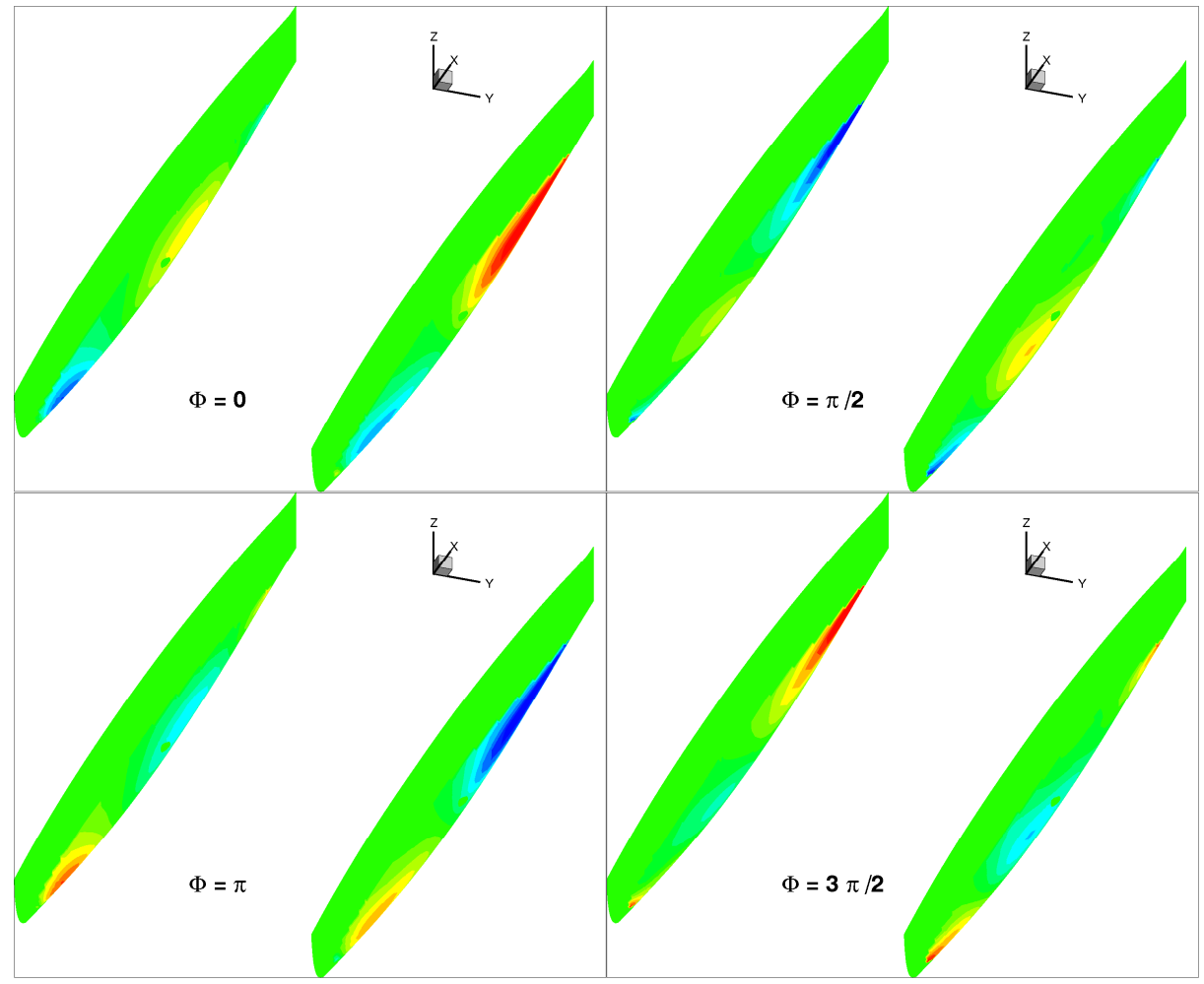

Figure 11. Pressions hydrodynamiques appliquées au modèle EF. 


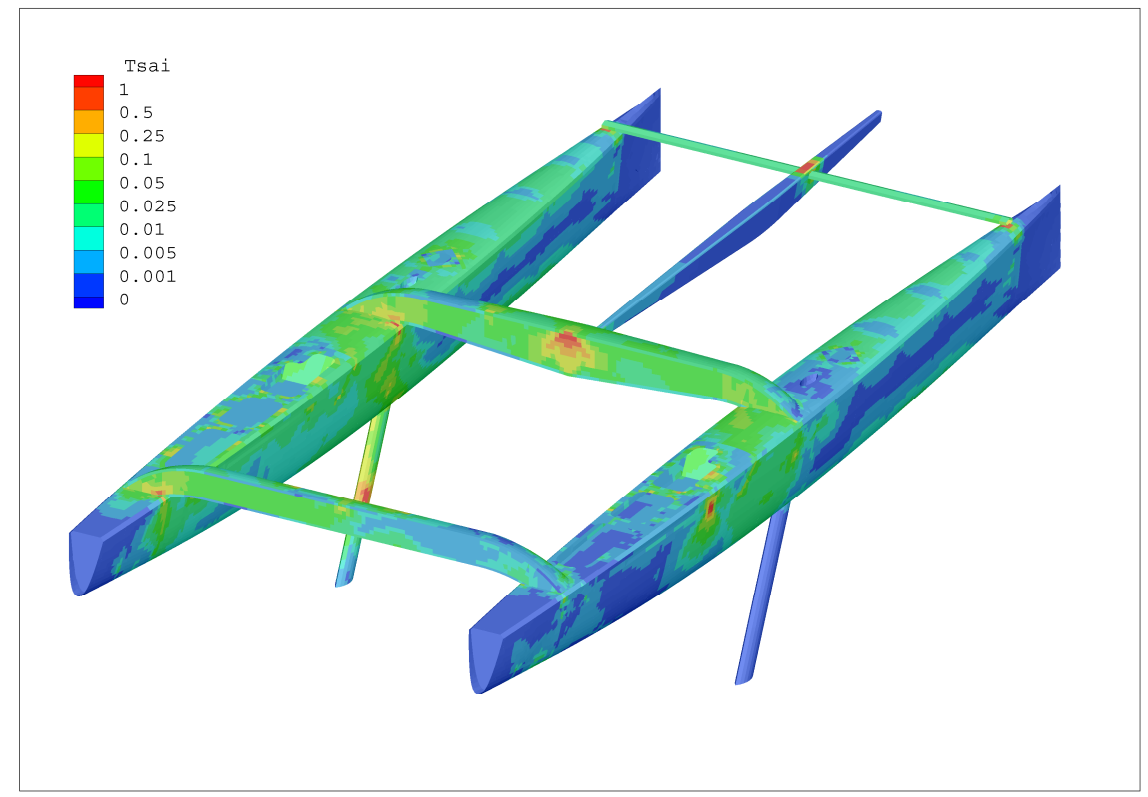

Figure 12. critère de Tsai sur le voilier lors de sa navigation dans la houle

\subsection{Les courses autour du monde}

Depuis 1993 ou Bruno Peyron a établi le record du tour du monde en descendant sous la barre des 80 jours (79 jours, 6 heurs et 16 minutes) sur le catamaran Commodore Explorer (ex Jet Service V), le record du trophée Jules-Verne n'a pas cessé de tomber. Ce record correspondait en 1993 à une vitesse moyenne de 14,39 nœuds (345,35 milles par jour). Depuis 1993, plusieurs skippers sur multicoques (trimaran ou catamaran) sont partis pour établir de nouveaux temps. En 1994, Peter Blake à sa première tentative bat le record en 74 jours et 22 heures et 17 minutes sur le Catamaran Enza de taille 22m. En 1997, Olivier de Kersauzon établit le record en 71 jours et 14 heures (Trimaran Sport Elec). Depuis 2002 Bruno Peyron est l'actuel détenteur du chrono sur le catamaran « code 0 » Orange $(33 \mathrm{~m})$ en 64 jours, 8 heures et 37 minutes et 24 secondes. On peut également souligner qu'Ellen Mac Arthur et Olivier de Kersauzon ont échoué dans cette course au chronomètre en 2002 respectivement sur un catamaran et un trimaran. Le code «B1 » présenté dans cette étude sera destiné aux grandes courses océaniques. L'échantillonnage de ces coques et de ces bras est constitué d'âmes Nomex® et de tissus carbone, dont le nombre de couches, leurs grammages et orientations sont évidemment confidentiels. Il convient cependant de noter que les résultats des nombreuses simulations menées à l'aide de 
la méthode des éléments finis ont conduit l'équipe à des renforcements locaux du voilier.

\section{Conclusions}

De nombreux travaux scientifiques concernant le comportement des nids d'abeilles Nomex® ont été réalisés afin de simuler le comportement mécanique homogénéisé de ces âmes. L'étude montre que l'approche retenue permet notamment de retrouver par des simulations éléments finis les contraintes ultimes de cisaillement fournies par les fabricants. L'influence de la hauteur de l'âme peut être vérifiée à l'aide de notre logiciel. La démarche retenue permet de calculer l'ensemble des contraintes ultimes et permet de créer une enveloppe de rupture de ces âmes. Les résultats issus de notre logiciel prototype «NidaCore» permettent de nourrir les modèles éléments finis tridimensionnels dédiés à l'étude du renforcement des voiliers multicoques. La validation des solutions retenues pour la conception d'un catamaran nécessite de mener à bien de nombreuses simulations éléments finis multiéchelles. La simulation de la réponse d'un catamaran dans la houle présentée dans la dernière partie montre une structure avec un critère de dimensionnement de Tsai homogène sur l'ensemble de la structure. Ces simulations nécessitent des données précises sur le comportement des âmes le constituant.

\section{Bibliographie}

Aminanda Y., Castanie B., Barrau J.J., Thevenet P., «Etude expérimentale et modélisation de l'indention des structures sandwichs à âme nid d'abeilles », JNC 13, Strasbourg, 2003, pp.461-470.

Bornert M., Bretheau T., Gilormini P., Homogénéisation en mécanique des matériaux 2, Paris, Hermès 2001.

Debordes O., «Homogenization computations in elastic or plastic range ; applications to unidirectional composites and perforated sheets », $4^{\text {th }}$ Int. Symp. Innovative Num. Method in Engng, Computational Mechanics Publications, Springer-Verlag, 1982, pp. 453-458.

Delhommeau G., Les problèmes de diffraction-radiation et de résistance de vague : étude théorique et résolution numérique par la méthode des singularités, ENSM 1988.

Det Norske Veritas, Rule for classification of high speed, light craft, July 1996.

Forest S., « Milieux continus généralisés et matériaux hétérogènes », Mines Paris Les Presses, 2006

Gibson L.J., Ashby M.F., «Cellular solids structures and properties », Pergamon Press, Oxford, 1988.

Gibson L.J., Ashby M.F., Shajer G.S., Robertson C.I., « The mechanics of two-dimensional cellular matériels », Proc. R. Soc. Lond, A382, 1982, pp. 25-42. 
Gornet L., Marckmann G., « Analyse par éléments finis du catamaran Code B1, plans Gilles Ollier Design Team », rapport interne LMM, 2003.

Gornet, S. Marguet, G. Marckmann, «Failure criteria and effective elastic properties of Nomex ${ }^{\circledR}$ honeycomb cores : modelling and experimental validation »International Journal Computer Material \& Continua,Tech science Press, 2006.

Gornet L., Royer J., Sablin S., «Prévision de la dégradation de composites carbone époxy stratifiés : application aux voiliers multicoques », JNC 12, Cachan France, pp. 51-59, 2000 .

Gornet L., «Comportement mécanique et analyse des structures en matériaux composites », cours Ecole Centrale Nantes 2001.

Grediac M., "A finite element study of the transverse shear in honeycomb cores », International Journal of Solids Structures, 30, 1993, pp.1777-1788.

Herakovich CT., « Mechanics of fibrous composite », John Wiley 1998.

Hohe J., Becker W., «A refined analysis of the effective elasticity tensor for general cellular sandwich cores », International Journal of Solids Structures, Elsevier, 38, 2001, pp.36893717.

Hohe J., Becker W., «An energetic homogenisation procedure for the elastic properties of general cellular sandwich cores », Composite Part B, 32, 2001, pp. 185-197.

Kelsey S., Gellatly R.A., Clark B.W., The shear modulus of foil honeycomb core, Aircraft Engng, 1958, pp.294-302.

Lene F., Contribution à l'étude des matériaux composites et de leurs endommagements, Thèse de Doctorat, Université Paris VI, 1984.

Nids d'abeilles Nomex ${ }^{\circledR}$, http://www.euro-composites.com

Shang J. F., Ashby M.F., «The Out-of-Plane properties of honeycombs », International Journal Mech. Science, Pergmon Press, 34(6), 1992, pp. 475-489.

Suquet P., Plasticité et homogénéisation, Thèse de Doctorat d'état, 1982.

The Cata, Course au Large, 8, 2003, pp. 78-81.

Verpeaux P., Charras T., Millard A., Castem 2000 : Une approche moderne du calcul des structures, JM Fouet, P Ladevèze, R Ohayon, 2, pp. 261-227, 1988. 